option is ideal, but might prove financially unrealistic.

(b) to rely on the recommendations of an individual sponsor, although objectivity regarding this method cannot be guaranteed

(c) to form a sponsoring organisation which consists of senior psychiatrists in the home country. This is part of the guidelines of the Royal College of Surgeons (Overseas Doctors Training Scheme, January 1989) which add that individual consultants and relatives of the candidate are not acceptable as sponsors.

Although I prefer the first option I opt for the last, due to its easy applicability.

I would welcome colleagues' suggestions and comments.

Springfield Hospital

London SW17 7DJ

\section{Dr Caldicott replies}

Professor Sims and I welcome Dr Abd's thoughtful letter concerning this training scheme, which constitutes part of the work of the Overseas Desk at the College (now renamed the Working Group for Overseas Liaison).

I should like to assure readers that we have been very careful to consider the points which Dr Abd so rightly raises. In particular, those overseas candidates who meet our criteria for acceptance onto the Scheme, which are stringent, currently have equal chances of placement on an approved UK training scheme. This may change if over-subscription occurs, when we will have to introduce a process of selection.

Ways and means by which selection of the best candidates can be made are currently being examined, and readers' views on how we could ensure that this occurs would be welcome. At the moment we have to leave the selection of suitable trainees to the overseas sponsors, who are carefully vetted. If mutual trust between the College and such a sponsor breaks down, e.g. if an unsuitable trainee is recommended, a sponsor will no longer be deemed acceptable either.

Ultimately, we may be able to hold "appointments committees" overseas, particularly if the question of financial support for the scheme, which is growing, is addressed constructively by the Department of Health. The College cannot afford to act as an em- ployment agency for Health Authorities to any great extent. Meanwhile, at least one Fellow of the College overseas in planning to conduct a competitive examination for potential trainees, which may be one way forward. However, it is important to note that the situation will vary according to the overseas country under consideration, as expectations and needs of the Scheme will vary among overseas sponsors and their trainees.

Finally, I should like to appeal to Tutors who have not, as yet, approached us with a view to taking an overseas trainee onto their scheme. We are running out of placements, and yes! the trainees can start their employment as an SHO.

Fiona CALdicott Sub-Dean Overseas Doctors Training Scheme

\section{Training experience in Australia}

DEAR SIRS

I read with interest the article 'An Australian Exchange' by Dr Harrison (Psychiatric Bulletin, July 1989, 13, 361-362).

I should like to point out that a one for one exchange (which is often difficult to arrange) is not necessary for overseas trainees who wish to have training experience in Australia. Instead, the Occupational Training Visa, arranged by the Australian Postgraduate Medical Federation is quite adequate; it is easily and rapidly obtainable and also enables trainees spouses (or companions) to work while here.

This hospital has developed a tradition of having one or two overseas trainees on its staff and it has been found to be a mutually rewarding experience.

I would suggest that trainees who may wish to spend a period in Australia should write to the hospital concerned enquiring about suitable vacancies. Psychiatric hospitals, many of which are university affiliated, have relatively larger numbers of trainees and thus tend to have more vacancies compared with the general hospital psychiatric units. I should be pleased to offer advice if needed.

Hillcrest Hosital

NORMAN JAMES Chief Executive Officer GPO Box 202

Adelaide

South Australia 5001 